Ann. Génét. Sél. anim., I977, 9 (3), 335-35I.

\title{
Amélioration génétique de la production laitière des brebis Sardes III. - Recherche d'une interaction
Bélier $\times$ Nombre de traites journalières
}

\author{
S. CASU, M. T. BENYOUCEF (*) et J.-C. FLAMANT $(*)$
}

Avec la collaboration technique de R. Carta et Suzanne Crochez (*)

Istituto Zootecnico e Caseario per la Sardegna

Bonassai Sassari (Italie)

(*) Station d'Amélioration Génétique des Animaux

Centre de Recherches de Toulouse, I.N.R.A., 31320 Castanet Tolosan

\begin{abstract}
Résumé
Les brebis, filles de 68 pères de race Sarde, ont été réparties au hasard, après sevrage des agneaux en 2 groupes, l'un trait 2 fois par jour, l'autre une seule fois. Les analyses de variance portant sur un total de I 058 lactations (528 premières, 440 secondes, et 90 troisièmes) ne mettent en évidence aucun effet significatif d'interaction entre le père et la fréquence de traite de ses filles sur les variables de production ou de teneur du lait. De même on n'observe pas d'écart significatif des coefficients d'héritabilité entre $x$ et 2 traites journalières bien que les valeurs obtenues avec une seule traite journalière soient généralement plus faibles.
\end{abstract}

\section{Introduction}

L'amélioration des performances laitières des brebis constitue un objectif prioritaire pour les populations ovines traites. Deux articles précédents (CASU et al., r975; Flamant et CASU, 1977) ont analysé les paramètres génétiques des critères productifs des brebis Sardes et fourni ainsi des éléments de raisonnement pour la mise en place d'un programme de sélection en race pure.

Mais la facilité de la traite doit constituer également un objectif important comme pour toutes les espèces laitières. Chez les brebis où les systèmes de traite mécanique en groupe (système CASSE, Bosc, I963; Bosc et al., r967) ou en continu (Morag et al., I974) constituent les solutions les mieux adaptées, le critère de facilité de traite le plus intéressant pour l'éleveur est la tolérance des 
brebis à supporter une simplification des opérations de traite (traite sans repasse) ou une réduction de la fréquence des traites ( $\mathrm{r}$ seule traite par jour ou suppression de la traite du dimanche) = Flamant, I973.

Par rapport aux autres races, la race Sarde présente l'intérêt essentiel de permettre la réalisation d'une seule traite par jour sans que la chute de production moyenne, par rapport aux brebis traites 2 fois par jour, dépasse I5 p. IOo (CASU et Boyazoglu, I973).

Cette valeur n'est cependant pas négligeable et la perte financière qui l'accompagne n'est pas forcément compensée par l'économie de main-d'œuvre. Il est donc intéressant d'étudier dans quelle mesure la prise en compte de ce caractère est possible dans un schéma de sélection. Les expériences de Classson et al. (I959) sur des couples de vaches jumelles monozygotes peuvent en effet laisser supposer l'existence d'une variabilité génétique pour l'écart de production entre les femelles traites $\mathrm{I}$ ou 2 fois par jour.

La préoccupation de la présente étude est donc la recherche de différences éventuelles en béliers pour l'écart observé entre les productions obtenues avec I ou 2 traites par jour en race Sarde. Dans l'hypothèse où certains éleveurs voudraient exploiter les possibilités de cette race en réduisant la fréquence des traites, il est également important de raisonner leur participation à un schéma de sélection dans lequel d'autres éleveurs continueraient à traire 2 fois par jour.

\section{Matériel et méthodes}

\section{r. - Origine des données}

Les données ont été recueillies au cours des Campagnes I971, I972 et I973 sur I 058 lactations de brebis de race Sarde $(528$ premières, 440 secondes et 90 troisièmes lactations). Les brebis sont issues de 68 pères différents, utilisés en principe un $\epsilon$ seule annéee, et proviennent de 4 troupeaux naisseurs appartenant à l'Istituto Zootecnico e Caseario per la Sardegna (Bonassai, Foresta Di Burgos, Macomer, MonASTIR). Quel que soit leur troupeau d'origine elles ont été rassemblées à l'âge de 6 mois et conduites ensuite en un seul troupeau à BonASSAI (CASU et al., 1975).

La traite à la machine est effectuée sans repasse (manuelle ou mécanique) après une période d'allaitement dont la longueur est fonction de l'âge de la mère (20 à 30 jours chez les antenaises et 30 à 45 jours chez les mères adultes).

\section{2. - Dispositif expérimental}

En première lactation, chaque groupe de filles $(n \geqslant$ ro) d'un bélier est divisé en deux sous-grouues, approximativement égaux $\left(n_{1}\right.$ ou $\left.n_{2} \geqslant 4\right)$ tirés au sort à la fin de la période d'allaitement.

L'un des deux sous-groupes est trait 2 fois par jour, l'autre une seule fois par jour le matin, la même fréquence de traite étant adoptée jusqu'au tarissement de chaque brebis. Ces groupes sont maintenus dans leur composition, aux disparitions près, au cours des lactations ultérieures, et traits avec la même fréquence journalière qu'en première lactation. Aucune sélection directe n'est exercée à l'issue de la première ou de la deuxième lactation, la diminution des effectifs étant due seulement aux mortalités et accidents divers (mammites par exemple). 


\section{3. - Variables analysées}

A partir des données de base enregistrées par le contrôle laitier, les variables suivantes ont été créées pour chaque lactation individuelle et soumises aux analyses statistiques :

a) Durée de la période de traite (jours).

b) Quantité de lait trait $(\mathrm{kg})$.

c) Production moyenne de lait par jour (g).

d) Quantité de matière grasse $(\mathrm{kg})$.

e) Taux butyreux $(\mathrm{g} / \%)$.

f) Quantité de matière azotée $(\mathrm{kg})$.

g) Taux azoté $(\mathrm{g} / \%)$.

h) Rapport taux azoté/taux butyreux (\%).

\section{4. - Analyses statistiques}

Un travail précédent (Flamant et CASU, I977) a montré l'incidence possible du troupeau de naissance des brebis sur leur production ultérieure dans l'élevage de Bonassar. Un facteur de classification global " Année de production - Troupeau de naissance " a donc été introduit dans toutes les analyses réalisées.

L'analyse des relations entre le génotype des brebis, représenté par le Bélier Père $(B)$, et leur Nombre de traites journalières $(N)$, a été réalisée selon deux approches différentes :

- signification statistique de l'interaction génotype $\mathrm{X}$ milieu (changement de rang des génotypes selon le milieu de production);

- changement de la variance génétique selon les milieux.

La signification et les modalités de ces 2 méthodes ont été résumées par BowMAN (I972).

a) Analyse de variance avec interaction.

Le modèle mixte suivant a été utilisé :

$\mathrm{Y}_{i j k l}=\mu+\mathrm{N}_{i}+\mathrm{AT}_{j}+(\mathrm{N} . \mathrm{AT})_{i j}+\mathrm{B}_{j k}+(\mathrm{N} . \mathrm{B})_{i j k}+e_{i j k l}$.

$\mathrm{N}_{i}=$ effet fixé du Nombre de traites $i(i=\mathrm{I}$ et 2$)$.

$\mathrm{AT}_{j}=$ effet fixé de l'Année-Troupeau $j(i=\mathrm{I}$ à 3$)$.

$(\mathrm{N} . \mathrm{AT})_{i j}=$ effet fixé d'interaction entre les deux facteurs précédents.

$\mathrm{B}_{j k}=$ effet aléatoire du Bélier $k$ utilisé dans l'Année-Troupeau $j$.

$(B . N)_{i j k}=$ effet aléatoire d'interaction entre les facteurs Béliers et Nombre de traites.

$e_{i j k l}=$ effet résiduel aléatoire.

Ce modèle général a été décomposé en deux sous-modèles :

$\mathrm{Y}_{i j l}=\mu+\mathrm{N}_{i}+\mathrm{AT}_{j}+(\mathrm{N} . \mathrm{AT})_{i j}+e_{i j l}$

$\mathrm{Y}_{i j k}=\mu+\mathrm{N}_{i}+\mathrm{B}_{k}+(\mathrm{B} . \mathrm{N})_{i k}+e_{i j k}$

ce dernier modèle étant valable pour chaque "Année-Troupeau " $j$.

La méthode de calcul et le tableau des espérances des carrés moyens sont détaillés en Annexe. 


\section{b) Estimation des coefficients d'héritabilité.}

Les coefficients d'héritabilité $h^{2}{ }_{1}$ et $h^{2}{ }_{2}$ des performances des brebis traites respectivement I fois et 2 fois par jour ont été estimés à partir des corrélations entre demi-sœurs paternelles pour chaque numéro de lactation.

Le modèle hiérarchique suivant a été utilisé

$\mathrm{Y}_{i j k}=\mu+(\mathrm{AT})_{i}+\mathrm{B}_{i j}+e_{i j k}$

$\mathrm{AT}_{i}=$ effet fixé de l'Année-Troupeau $i$

$\mathbf{B}_{i j}=$ effet aléatoire du Bélier $j$ utilisé dans l'Année-Troupeau $j$

$e_{i j k}=$ effet aléatoire résiduel.

\section{Résultats}

\section{I. - Effets non génétiques}

Le tableau I rapporte les quantités de lait moyennes obtenues pour les différents lots au cours des 3 campagnes expérimentales. Pour simplifier la présentation, nous nous sommes contentés d'indiquer dans le tableau 2 les différences entre les performances moyennes des brebis traites I et 2 fois pour les 8 variables considérées. Les tableaux 3, 4 et 5 donnent les résultats des tests de signification statistique pour les différentes analyses de variance réalisées avec interaction.

L'effet combiné de l'année de production et du troupeau de naissance est le plus souvent significatif sur la plupart des performances. Cependant les phénomènes d'interaction $(\mathrm{N} \times \mathrm{AT})$ ne sont pas très importants.

L'effet du nombre de traites journalières se manifeste de la façon suivante sur le niveau des performances :

\section{Durée de traite.}

La différence moyenne pour l'ensemble des lactations contrôlées n'atteint pas 3 jours et est de surcroît en faveur des brebis traites une seule fois par jour. La réalisation d'une seule traite quotidienne ne s'accompagne donc pas d'un raccourcissement de la durée de lactation.

\section{Niveaux de production.}

On observe un écart de production de l'ordre de I4,6, Ir,5 et 6,2 p. Ioo par rapport aux brebis traites 2 fois par jour, respectivement en première, deuxième et troisième lactations, soit une perte moyenne de I0,7 p. roo pour l'ensemble des lactations contrôlées. La comparaison des résultats obtenus sur les lots ayant eu 2 lactations successives ( $I^{\text {res }}$ lactations en I97I et I972) révèle que l'écart moyen de production entre les 2 fréquences de traite reste à peu près constant en valeur absolue et apparaît donc relativement plus faible lorsque le niveau de production augmente avec l'âge. Le même pourcentage de perte et la même tendance à une diminution avec le numéro de lactation se manifestent pour la quantité moyenne journalière, les quantités de matière grasse et matière azotée. 


\section{Teneurs du lait.}

Un fait intéressant qui mérite d'être souligné concerne la modification du rapport des taux. La simplification de la traite semble, en effet, avoir des conséquences différentes sur chacun des 2 taux considérés (tabl. I) $=$ le taux butyreux subit une baisse moyenne de $3 \mathrm{p}$. Ioo alors que le taux azoté se trouve légèrement augmenté en moyenne de I p. Ioo. Il en résulte que le rapport taux azoté/taux butyreux est supérieur de 2 à 4 p. Ioo sur les lots de brebis traites une seule fois par jour.

\section{2. - Interaction et effet du nombre de traites sur le niveau d'héritabilité}

Le mode d'utilisation des béliers (non répétition d'une année à l'autre et d'un troupeau naisseur à l'autre) fait que les facteurs "Année-Troupeau " (AT) et "Béliers" (B) sont hiérarchiques. Il en résulte que le niveau de l'interaction "AnnéeTroupeau $\mathrm{X}$ Nombre de traites " $(\mathrm{AT} \times \mathrm{N})$ dépend largement d'une éventuelle interaction "Bélier $\mathrm{X}$ Nombre de traites " $(\mathrm{B} \times \mathrm{N})$. L'absence totale d'une interaction significative " $\mathrm{B} \times \mathrm{N}$ ) que nous observons ici constitue donc une confirmation de l'absence d'interaction " $\mathrm{AT} \times \mathrm{N}$ » (tabl. 3, 4 et 5). En revanche l'effet "Bélier " est hautement significatif sur la plupart des variables en première et en deuxième lactations.

Les héritabilités calculées sur les performances obtenues avec 2 traites quotidiennes sont dans la plupart des cas plus élevées notamment pour la quantité de lait et le taux butyreux en première lactation (tabl. 6 et 7 ).

C'est ainsi que l'on observe des valeurs de variance génétique jusqu'à 2 fois plus élevées dans le cas de 2 traites tandis que le rapport de variances phénotypiques 2 traites / I traite est au plus de I,5. Cependant les effectifs concernés sont trop faibles pour permettre la mise en évidence de différences significatives entre valeurs des coefficients d'héritabilité.

\section{Discussion}

Les écarts de production observés entre les 2 fréquences de traite journalière (I et 2 fois par jour) sur notre échantillon sont identiques à ceux estimés par CASU et Boyazoglu (I973) sur l'ensemble des lactations réalisées dans le troupeau de BonassaI au cours de ces mêmes années d'expériences (I 4 II lactations) $=$ I4 à I6,6 p. Ioo pour les agnelles, I5 p. Ioo pour les brebis en deuxième lactation et Ix,9 p. Ioo pour les adultes.

Comme l'indiquent déjà ces mêmes auteurs, ces valeurs sont très faibles comparées aux résultats obtenus sur d'autres races ovines ou sur d'autres espèces. Ainsi LABUssière et al. (I974), sur des brebis de race Préalpes $d u$ Sud, comparables aux brebis Lacaune, observent une perte nettement plus importante (environ $50 \%$ ) avec la suppression d'une traite par jour. Flamant (I973) obtient une perte de I9 p. Ioo sur des brebis Lacaune traites une seule fois par jour, supérieure aux I5 p. Ioo observés sur des brebis Sardes, la suppression d'une traite journalière intervenant en fin de lactation. Mocouor et AURAN (I974) relèvent des diminutions relativement importantes sur 83 observations de chevrettes croisées de type Alpin (Chamoisée $\times$ Saanen) de même ordre que celles enregistrées chez les bovins 


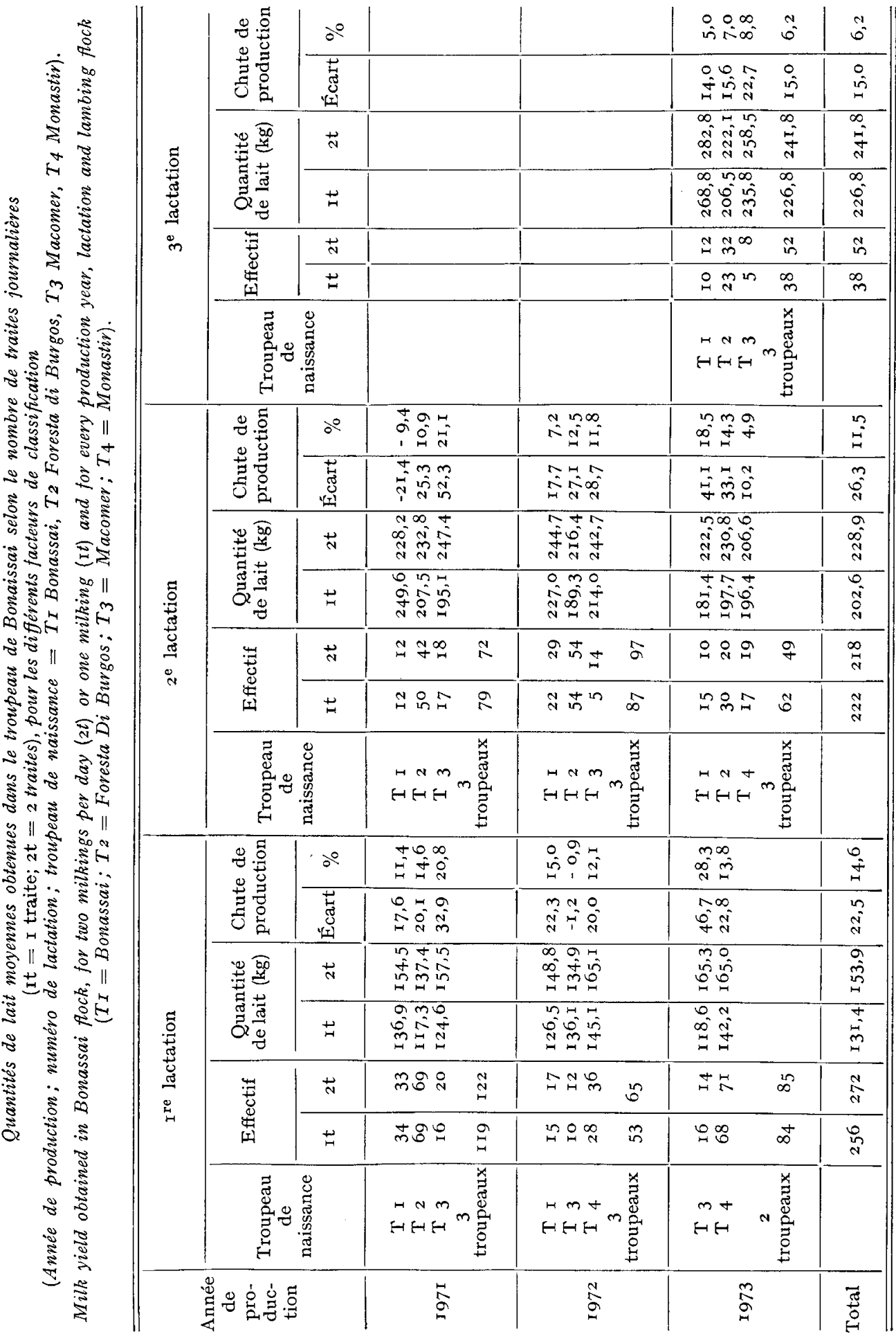




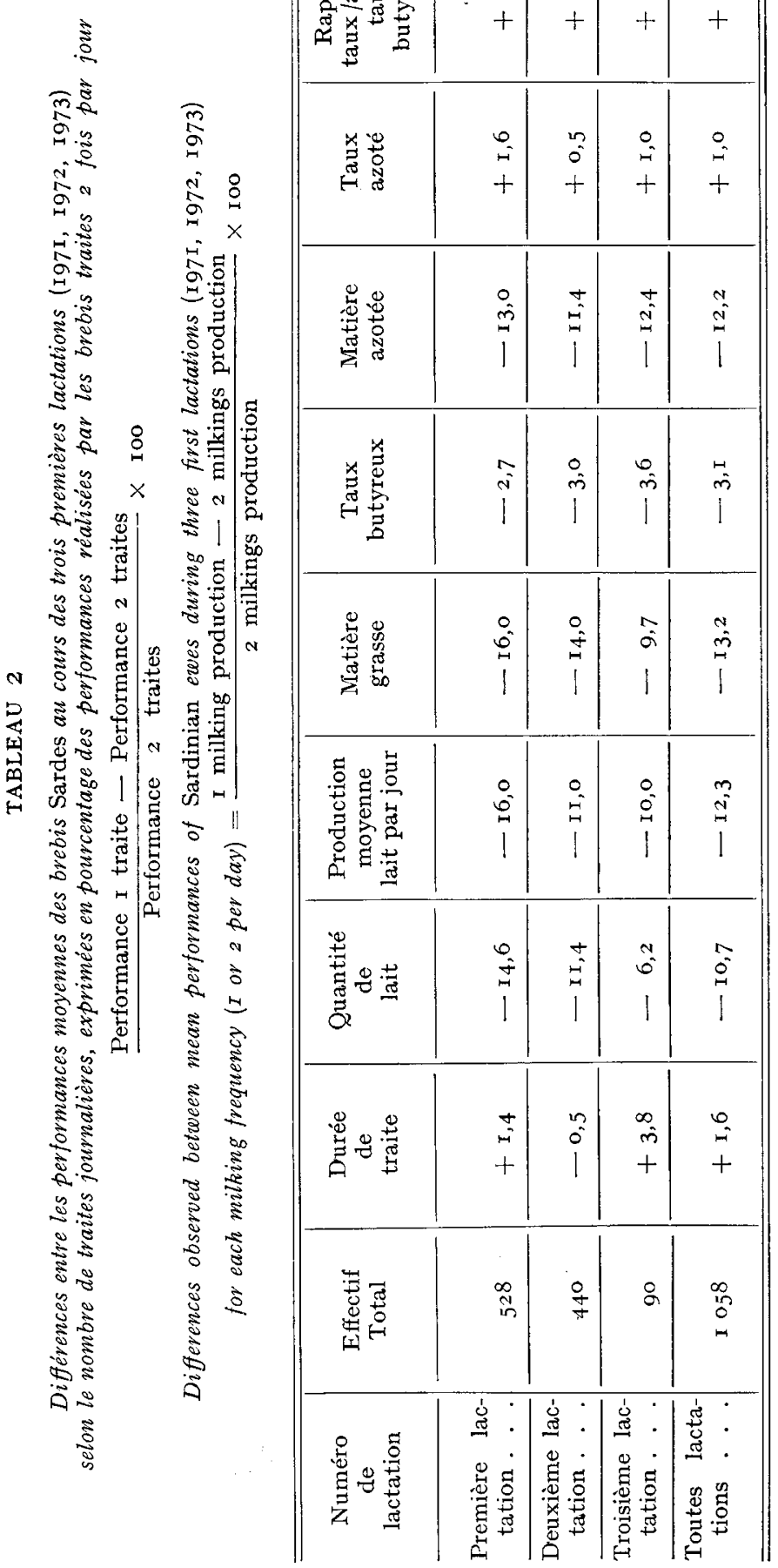




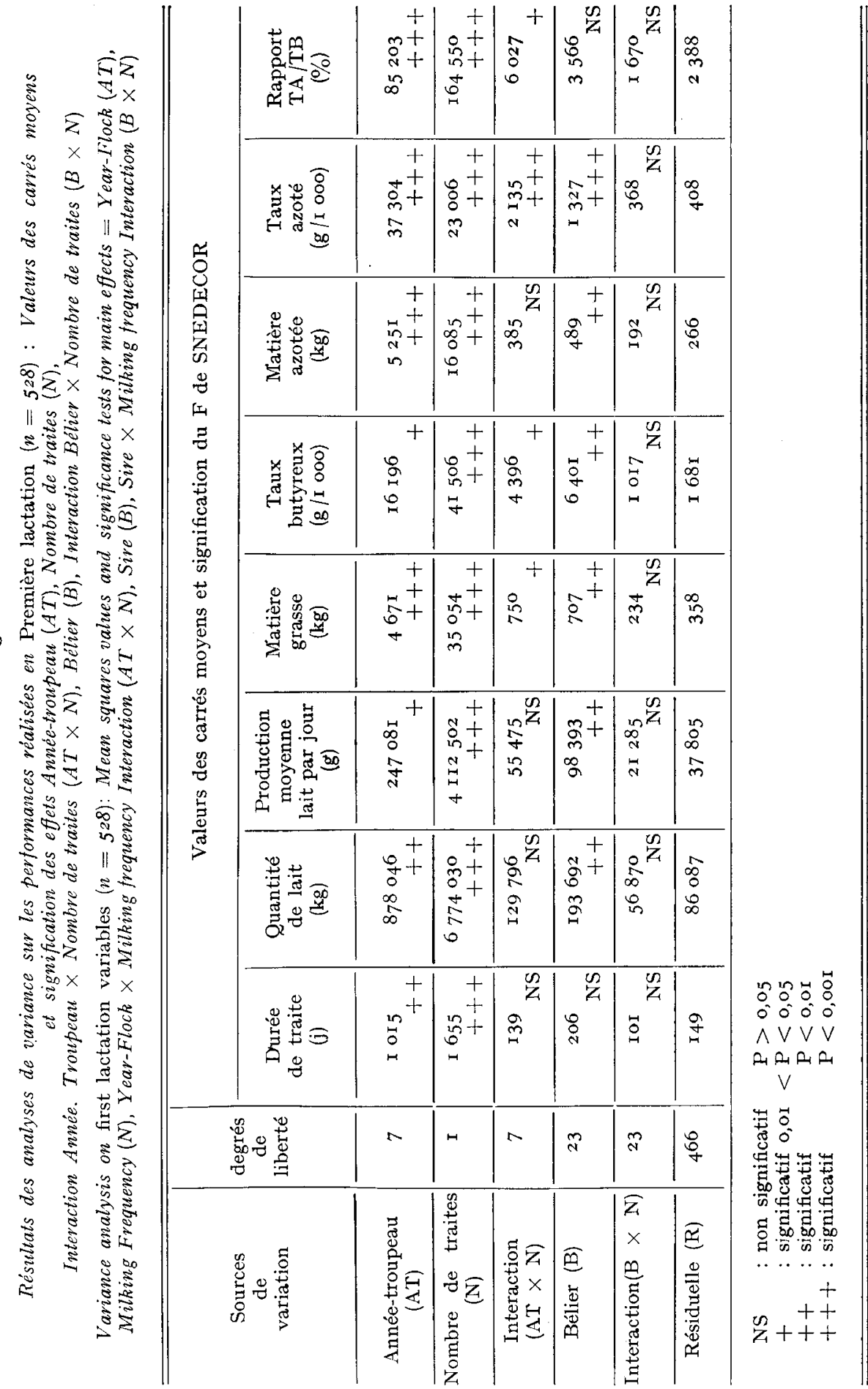




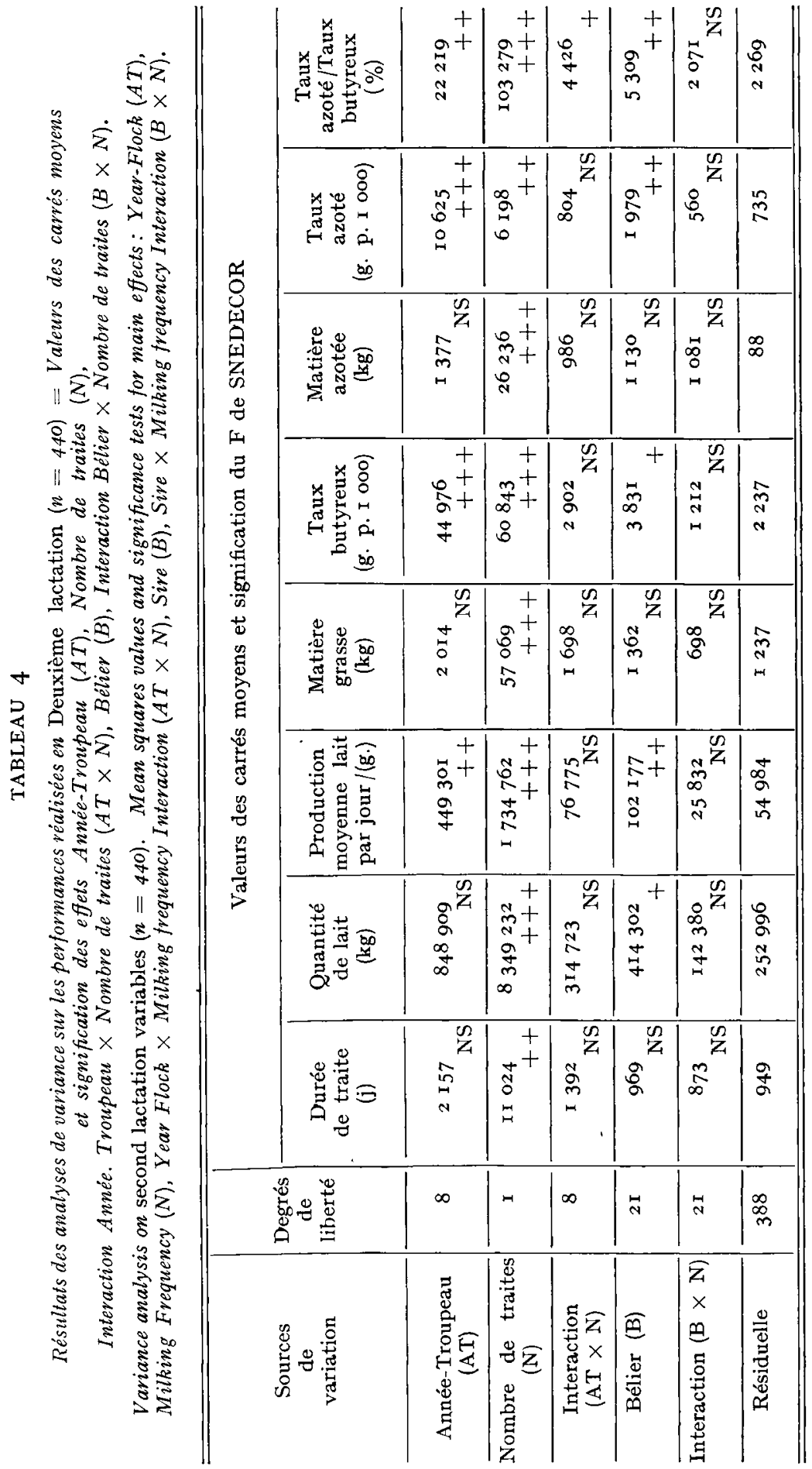




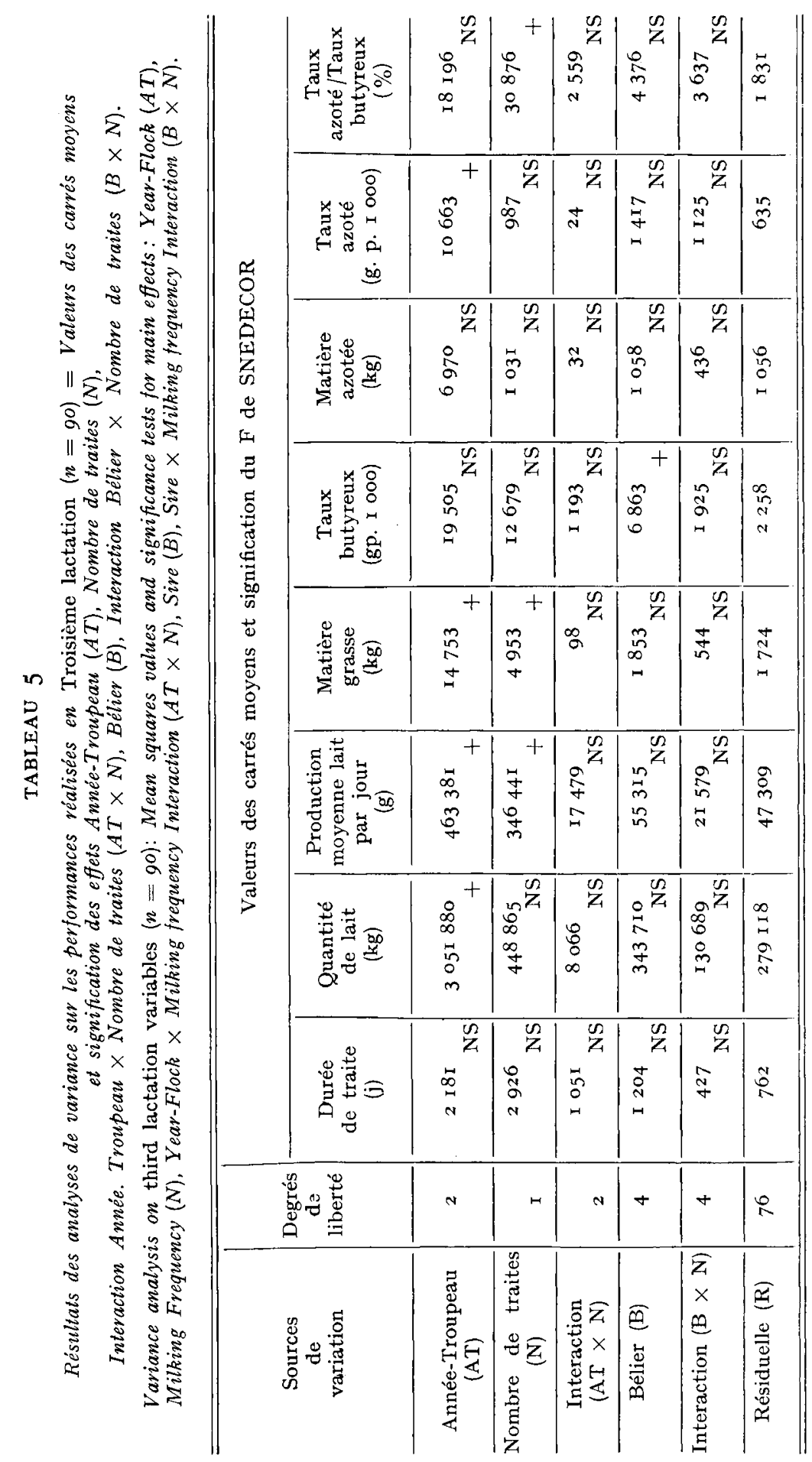




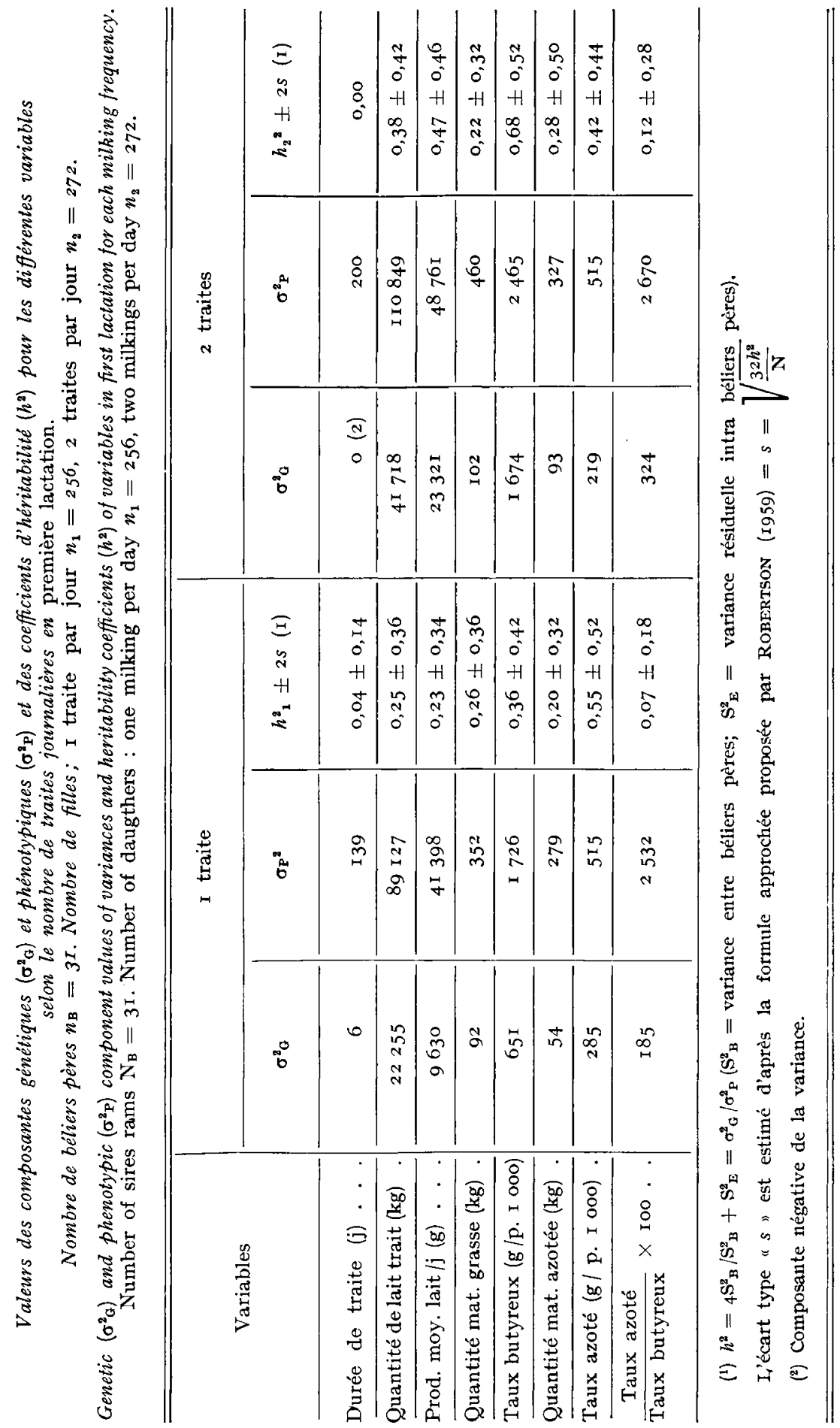




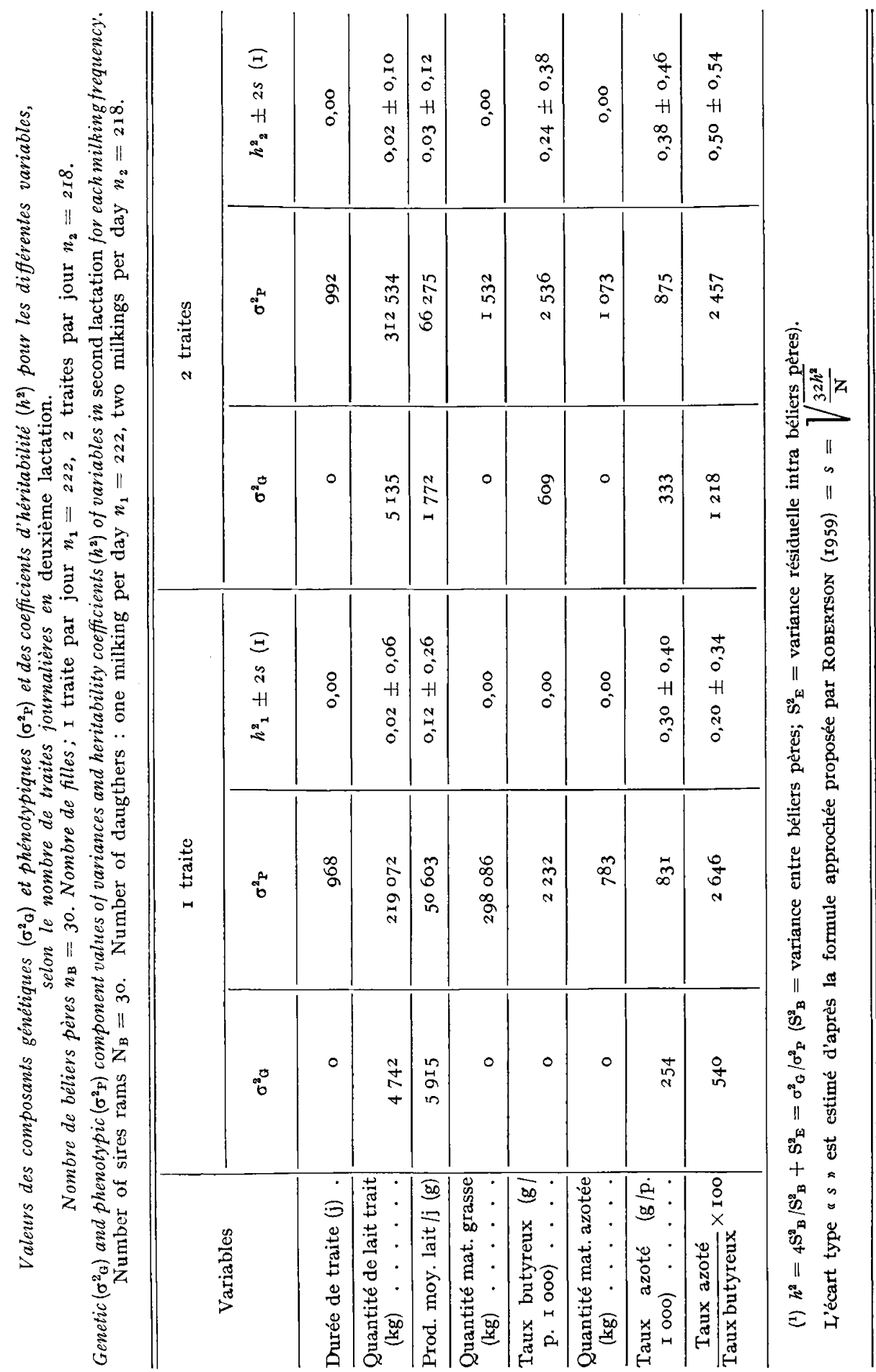


par Woodward (I93I) (56\%) et par CLAEsson et al. (I959) qui signalent également une perte moyenne de $50 \mathrm{p}$. Ioo en première lactation. MoraG (1968) effectuant une expérience sur deux lots de 6 brebis, compare les productions des demimamelles traites I, 2 et 3 fois par jour. Dans un lot, la traite est suivie de deux injections d'ocytocine pour extraire le lait résiduel. Il observe ainsi un écart de $20 \mathrm{p}$. Ioo entre les productions des demi-mamelles traites I et 2 fois par jour.

Il se confirme que la race Sarde supporte la pratique d'une seule traite journalière avec une chute de production plus faible que pour les autres races ovines et les autres espèces laitières. On peut supposer que cette supériorité sur les autres races ou espèces laitières est due à des caractéristiques anatomiques (citerne volumineuse, meilleure souplesse de la mamelle) ou physiologiques qu'il serait intéressant d'analyser. Ce comportement très particulier de la race Sarde constitue vraisemblablement un caractère racial très important dont la souche synthétique F.S.L. tente de réaliser l'utilisation conjointement aux qualités des races Lacaune et Frisonne (Flamant, I974).

La réalisation d'une seule traite quotidienne entraîne également une modification de la production de matière sèche. Les pertes enregistrées pour la quantité de matière grasse sont légèrement supérieures à celles observées pour la quantité de matière azotée sauf en troisième lactation. Comme pour la quantité de lait, ces valeurs sont nettement inférieures à celles obtenues par ClaEsson et al. (I959) sur vaches laitières où l'écart atteint $57 \mathrm{p}$. Ioo.

En revanche l'ensemble des résultats obtenus par les différents auteurs est cohérent pour la teneur du lait $=$ elle n'est que peu modifiée par la fréquence des traites avec cependant une légère tendance à l'augmentation du taux azoté.

On peut se demander pourquoi les interactions (Béliers $\times$ Nombre de traites des filles) ne sont jamais significatives alors que ClaEsson et al. (I959) observent au contraire des écarts de performances significatifs selon le couple de vraies jumelles concerné. Les différences relativement faibles observées entre lots traits I fois et 2 fois par jour sur brebis Sardes par rapport à d'autres races ou espèces ne sont peut-être pas suffisantes pour induire une interaction génotype $\times$ milieu. Pour confirmer cette hypothèse, il serait intéressant de travailler avec des races telles que la Lacaune, beaucoup plus sensibles à un changement de la fréquence des traites. Ceci signifierait également que la sélection sur la facilité de traite risque d'être d'autant plus efficace (et intéressante) que les conditions de traite sont difficiles (simplification des opérations de traite ou réduction de la fréquence des traites). Aucune expérimentation n'est actuellement réalisée qui permettrait de conclure sur ces différents points.

Cette même absence d'interaction avait été observée précédemment sur la quantité de lait lors d'une analyse préliminaire réalisée par Flamant et al. (I973) avec un modèle statistique légèrement différent et sur un échantillon de lactations plus réduit. Par rapport à cette première analyse, les coefficients d'héritabilité sont cependant plus faibles du fait que l'on a pris en compte ici le troupeau d'origine des brebis dans le modèle d'analyse (FLamant et CASU, I977). On observe par ailleurs que les estimées obtenues avec 2 traites par jour sont légèrement supérieures à celles obtenues avec I seule traite, bien que ces différences ne soient pas significatives. Ce résultat, s'il était confirmé, pourrait s'expliquer par le fait que la réalisation d'une seule traite par jour constitue un facteur beaucoup plus limitant pouı les brebis à potentiel de production élevé que pour les autres. Ceci contribuerait donc à limiter la variabilité génétique du niveau de production laitière. FimLand et al. (1972) observent sur vaches Frisonnes des valeurs d'héritabilité légèrement 
supérieures pour les productions obtenues avec 2 traites par jour par rapport à 3 traites journalières, mais ne donnent pas d'interprétation à ce résultat.

Du point de vue du testage des béliers de race Sarde sur leur descendance, et dans la mesure où les résultats présents seraient confirmés, les conclusions pratiques suivantes peuvent être retenues :

- le classement de la valeur génétique des béliers est le même, quel que soit le nombre de traites journalières de leurs filles. Il apparaît donc difficile d'envisager ici la possibilité d'une sélection ayant pour objectif la réduction de l'écart moyen entre les performances de production réalisées avec $r$ et 2 traites par jour;

- dans l'hypothèse où des éleveurs adopteraient la fréquence d'une seule traite journalière pour limiter les contraintes de travail ou accroître l'effectif de leur troupeau, on pourrait enregistrer une précision de testage plus faible et un progrès génétique moindre sur la quantité totale de lait (coefficients d'héritabilité plus faibles). Si le nombre de troupeaux contrôlés était par ailleurs suffisant, on pourrait donc juger préférable de ne considérer ces troupeaux à la traite simplifiée que comme des troupeaux commerciaux de multiplication. La conclusion serait totalement différente si l'on mettait en évidence une sélection plus efficace dans les conditions de traite les moins laborieuses.

\section{Conclusion}

Les résultats obtenus ici ne permettent pas de conclure à l'existence d'une interaction entre le génotype des brebis, représenté par leur père, et le nombre de leurs traites journalières. On peut en déduire que sur le présent échantillon n'apparaît pas une possibilité de sélection sur la facilité de traite exprimée par la différence de production obtenue avec I ou 2 traites journalières. Ceci n'est peut-être guère étonnant en race Sarde dont les aptitudes sont déjà exceptionnelles parmi les espèces et races laitières. Des expérimentations similaires réalisées sur des animaux où l'intérêt d'une telle amélioration génétique serait plus grand sont probablement trop coûteuses pour être réalisées commodément. Le coefficient d'héritabilité légèrement plus faible obtenu avec I traite par jour pourrait cependant signifier, si ce résultat était confirmé, que la diminution de la fréquence des traites s'accompagne de contraintes supplémentaires pour l'expression du caractère " production laitière ". Une sélection sur la production laitière dans ces conditions pourraient donc avoir pour signification à la fois une sélection sur les aptitudes de production laitière et sur les caractères héréditaires déterminant une réponse positive à ces contraintes. Tel pourrait être le cas par exemple de la souplesse de la mamelle qui limiterait la pression intra-mammaire à un seuil tel que la sécrétion lactée ne soit pas inhibée.

Ręu pour publication en juillet 1977.

\section{Remerciements}

Le modèle statistique utilisé dans cette analyse a été élaboré avec les conseils de J. RAzunGLes (Station d'Amélioration Génétique des Animaux, C. R. INRA, Toulouse).

Nous tenons à le remercier ici pour son utile contribution.

J. Boyazoglu, J. Labussière et J.J. Lauvergne ont assuré la lecture du manuscrit. 


\section{Summary}

\section{Genetic improvement of milk production of Sardinian ewes. II. Searching Ram $\times$ number of daily milkings interaction}

Inside progeny-groups from 68 sires of Sardinian breed, daughters are distributed at random in 2 lots after weaning ( 20 to 30 days for 1 st lambing ewes, 30 to 45 days for mature ewes). The two lots differ for the number of daily milkings: one or two time per day during the milking period. Inside every progeny-group the distribution is approximately equal between the two experimental lots.

We observe a production level for the " one milking" lot $\mathrm{I}_{4}, 6$ I 5,5 and 6,2 per cent lower than for the " two milkings" lot respectively in first, second and third lactation $(n=528,440$, 9o; tabl. $x$ and 2). No differences appear for the duration of the milking period. Milk composition is few modified: diminution of fat percentage, increasing of the protein one (tabl. 2).

These results demonstrate another time a very low dependance of Sardinian ewes from the milking frequency in respect to figures observed in other sheep breeds (LABUSsIìre et al., 1974) or in other species (ClaEsSON et al., 1959 for cows; Mocouot and AURAN, I974 for goats).

There is no evidence, from the results of variance analysis (tabl. 3, 4, 5) for interaction effect "Sire X milking frequency of the daughters", on milk yield or fat or protein percentage.

We do not observe significative differences between heritability coefficients estimated with $x$ or 2 milkings per day (tabl. 6 and 7). However, heritability values obtained with I milking per day are generally the lowest. For example, in first lactation and for total milk yied, the following values are estimated:

$$
\begin{array}{ll}
h^{2} \text { I milking }=0,25 \pm 0,36 \\
h^{2} \quad 2 \text { milkings }=0,38 \pm 0,42
\end{array}
$$

At the end of the paper, consequencies of these results are discussed by reference to the objectives of the selection in the Sardinian breed, as for the organisation of the progeny-testing in commercial flocks when their milking frequency differs.

\section{Références bibliographiques}

Bosc J., 1963. Problèmes de l'organisation du travail dans un troupeau de brebis laitières. Adaptation de la méthode "Avête de poisson" à la traite mécanique des brebis. Compte rendu à l'A.F.Z., 3-39.

Bosc J., Flamant J. C., Ricordeau G., 1967. Traite à la machine des brebis. Suppression de l'égouttage manuel ou remplacement par un égouttage machine. Ann. Zootech., 16 (2), 191-202. Bowman J. C., 1972. Génotype $\times$ environment interactions. Ann. Génét. Sél. Anim., 4 (I), II 7-r23.

Casu S., Boyazoglu J. G., 1973. Effet de la suppression de la traite du soir chez les brebis Sarde. Symposium International sur la traite mécanique des Petits Ruminants. Millau. 7-I I mai I973. Ann. Zootech., $\mathrm{n}^{0}$ hors série, 1974, I39-I44.

Casu S., Carta R., Flamant J. C., 1975. Amélioration génétique de la production laitière des brebis de race Sarde. I/Héritabilités et corrélations entre caractères. Ann. Génét. Sél. Anim., 7 (I), 73-90.

Clamsson O., Hansson A., Gustafsson N. et Brannang E., 1959. Studies on Monozygous Cattle Twins. XVII. Once - a - day milking compared with twice - a - day milking. Acta. Agric. Scand., 9, 38-58.

Fimland E. A., Bar Anan R., Harvey W. R., I972. Studies on dairy records from Israeli. Friesian cattle. II. Estimates of components of variance (covariance) and of parameters related to these components. Acta Agric. Scand., 22, 49-62..

Flamant J. C., I973. Quelques éléments de comparaison de l'aptitude à la traite des brebis des races Sarde et Lacaune élevées dans la région de Roquefort. Symposium International sur la traite mécanique des Petits Ruminants. Millau 7-II mai 1973. Ann. Zootech. No hors série. 1974. I63-I69. 
Flamant J. C., Rastogi R., Casu S., I973. Interaction bélier $\times$ nombre de traites journalières sur la production laitière des brebis Sardes (Résultats préliminaires). Symposium International sur la traite mécanique des Petits Ruminants. Millau 7-I I mai I973. Ann. Zootech. No hors série. r974. I7 I-I 73.

Flamant J. C., I974. Performances des brebis croisées en vue de la production laitière à la traite. I er Congrès mondial de génétique appliquée à l'élevage animal. Madrid 7-II oct. I974, 3, 973-977.

Flamant J. C., Casu S., I977. Amélioration génétique de la production laitière des brebis Sardes. II. Facteurs de variations génétiques et non génétiques des performances de brebis ayant réalisé 2 lactations. Ann. Génét. Sél. anim., 9, 203-2I 7 .

Labussière J., Combaud J. F., Petrequin P., 1974. Influence de la fréquence des traites et des tétées sur la production laitière des brebis Préalpes du sud. Ann. Zootech., 23, 445-457.

Mocouot J. C., Auran, 1974. Effets de différentes fréquences de traite sur la production laitière des caprins. Ann. Génét. Sél. anim., 6, 463-476.

MoRAG M., I968. The effect of varying the daily milking frequency on the milk yield of the ewe and evidence in the nature of the inhibition of milk ejection by half-udder milking. $A n n$. Zootech., $17,35 \mathrm{I}-369$.

Morag M., Sagi R., Rubin E., Arnon Y., Levy M., 1974. A proposal for a constantly rotating carousel for sheep milking (Projet de traite des brebis à l'aide d'un caroussel à rotation continue). Ann. Zootech., 23, 48I-489.

RoBertson A., 1959. Experimental design in the evaluation of genetic parameters. Biometrics, 15, $219-226$.

WOODWARD T. E., I93x. The production of dairy cows as affected by frequency and regularity of milking and feeding. U.S. Dept. Agric. Cir., r8o, I-16.

\section{Annexe}

Analyse de variance avec interaction

Méthode de calcul et tableau

des espérances mathématiques des carrés moyens

\begin{tabular}{|c|c|c|c|c|c|c|c|c|}
\hline \multirow{2}{*}{ 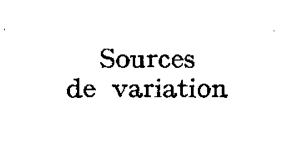 } & \multirow{2}{*}{$\begin{array}{l}\text { Nombre } \\
\text { de degrés } \\
\text { de liberté }\end{array}$} & \multirow{2}{*}{$\begin{array}{l}\text { Carrés } \\
\text { moyens }\end{array}$} & \multicolumn{6}{|c|}{ Espérances mathématiques } \\
\hline & & & $\sigma^{2} e$ & $\sigma^{2} \mathrm{B.N}$ & $(\mathrm{AT} \times \mathrm{N})^{{ }^{2} i j}$ & $\sigma^{2} B$ & $\left(\mathrm{AT}_{j}\right)^{2}$ & $(\mathrm{Ni})^{2}$ \\
\hline 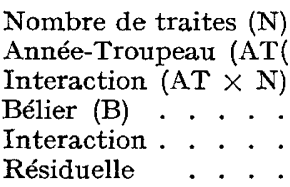 & $\begin{array}{c}p-\mathrm{I} \\
q-\mathrm{I} \\
(p-\mathrm{I})(q-\mathrm{I}) \\
q(r-\mathrm{I}) \\
q(p-\mathrm{I})(r-\mathrm{I}) \\
p q r(n-\mathrm{I})\end{array}$ & $\begin{array}{l}\mathrm{CM}_{\mathrm{N}} \\
\mathrm{CM}_{\mathrm{AT}} \\
\mathrm{CM}_{\mathrm{AT} \cdot \mathrm{N}} \\
\mathrm{CM}_{\mathrm{B} / \mathrm{AT}} \\
\mathrm{CM}_{\mathrm{B} . \mathrm{N} / \mathrm{AT}} \\
\mathrm{CM}_{\mathrm{E}}\end{array}$ & $\begin{array}{l}\text { I } \\
\text { I } \\
\text { I } \\
\text { I } \\
\text { I } \\
\text { I }\end{array}$ & $n$ & + & $p n$ & + & + \\
\hline
\end{tabular}

Soit

$$
\mathrm{C}=\frac{\mathrm{Y}^{2} \ldots}{p q r n} \quad \text { et } \quad \mathrm{T}=\sum_{i j k l} \mathrm{Y}^{2} i j k l
$$


On a alors les valeurs suivantes pour les différentes sommes de carrés centrés :

$$
\begin{aligned}
\mathrm{SCE}_{i j k} & =\sum_{l=1}^{n} \mathrm{Y}^{2} i j k l-\mathrm{Y}^{2} i j k \cdot l n \\
\mathrm{SCE}_{\mathrm{E}} & =\sum_{i} \sum_{j} \sum_{k} \mathrm{SCE}_{i j k} \\
\mathrm{SCE}_{\mathrm{N}} & =\frac{\mathrm{I}}{q r n} \sum_{i} \mathrm{Y}^{2} \ldots-\mathrm{C} \\
\mathrm{SCE}_{\mathrm{AT}} & =\frac{\mathrm{I}}{p r n} x \sum_{j} \mathrm{Y}^{2} \cdot j \ldots-\mathrm{C} \\
\mathrm{SCE}_{\mathrm{AT} . \mathrm{N}} & =\frac{\mathrm{I}}{r n} \sum_{i} \sum_{j} \mathrm{Y}^{2} i j \ldots-\mathrm{C}-\mathrm{SCE}_{\mathrm{N}}-\mathrm{SCE}_{\mathrm{AT}} \\
\mathrm{SCE}_{\mathrm{B}} & =\frac{\mathrm{I}}{p q r} \sum_{k} \mathrm{Y}^{2} \ldots k .-\mathrm{C} \\
\mathrm{SCE}_{\mathrm{B} . \mathrm{N}} & =\frac{\mathrm{I}}{q n} \sum_{i} \sum_{k} \mathrm{Y}^{2} i . k .-\mathrm{C}-\mathrm{SCE}_{\mathrm{N}}-\mathrm{SCE}_{\mathrm{B}} \\
\mathrm{SCE}_{\mathrm{AT} . \mathrm{B}} & =\frac{\mathrm{I}}{p n} \sum_{j} \sum_{k} \mathrm{Y}^{2} \cdot j k .-\mathrm{C}-\mathrm{SCE}_{\mathrm{AT}}-\mathrm{SCE}_{\mathrm{B}} \\
\mathrm{SCE}_{\mathrm{N} . \mathrm{AT} . \mathrm{B}} & =\mathrm{SCE} t-[(\mathrm{I})+(2)+(3)+(4)+(5)+(6)+(7)] \\
\mathrm{SCE}_{t} & =\mathrm{T}-\mathrm{C}
\end{aligned}
$$

L'analyse peut être effectuée en utilisant le schéma général et les formules suivantes :

$$
\begin{aligned}
& \mathrm{SCE}_{\mathrm{B}} / \mathrm{AT}=\mathrm{SCE}_{\mathrm{B}}+\mathrm{SCE}_{\mathrm{AT} . \mathrm{B}} \\
& \mathrm{SCE}_{\mathrm{B} . \mathrm{N} / \mathrm{AT}}=\mathrm{SCE}_{\mathrm{B} . \mathrm{N}}+\mathrm{SCE}_{\mathrm{N} . \mathrm{AT} . \mathrm{B}}
\end{aligned}
$$

